\title{
Il giornalismo etico: un'ispirazione per un tipo di comunicazioni responsabile in Europa
}

\section{Aidan White}

\section{INTRODUZIONE}

In un'epoca in cui la cittadinanza umana e l'empatia della società scarseggiano in gran parte dell'Europa, l'esigenza di civiltà nei dibattiti pubblici non è mai stata più grande. In alcuni Paesi, quali l'Ucraina e la Russia, si combattono vere e proprie guerre dell'informazione alimentate dalla propaganda nazionalista come se fossero battaglie per la conquista di territori e le politiche divisive del post guerra fredda continuano a dominare l'agenda mediatica. In altri Paesi i media, sempre più una sorta di trofei detenuti da personaggi ricchi e potenti, vengono strumentalizzati per promuovere gli interessi politici o economici di questi ultimi, con scarso riguardo alle finalità del giornalismo e all'interesse pubblico in senso più ampio. Questo turbolento panorama mediatico è chiamato ad affrontare un'ulteriore sfida in seguito all'avanzare di internet, che ha fortemente aumentato l'ambito della libertà, ma ha al contempo aperto le porte a forme comunicative sregolate, sfrenate e spesso violente.

In questo contesto, il giornalismo etico, le cui radici affondano nella nascita dei mass media circa 150 anni fa, rappresenta una vecchia concezione, la quale si sta, tuttavia, rivelando particolarmente importante nel porre le condizioni per la creazione di un tipo comunicazioni responsabile in Europa.

Il giornalismo etico consiste in una cornice finalizzata a fornire informazioni affidabili, accurate e rilevanti e dipende dalla capacità dei caporedattori dei giornalisti di pensare e di agire in modo indipendente nel fornire informazioni e notizie. Non può esistere se mancano la trasparenza, il pluralismo e professionalità dalla cima al fondo della piramide dei media. Ma queste condizioni scarseggiano nell'ambiente mediatico moderno. In tutta Europa le pressioni economiche e politiche dominano nelle redazioni ed i giornalisti fanno fatica a esprimere le loro voci in un contesto di valori.

In tutti i Paesi, i cambiamenti improvvisi e le crisi finanziarie causate dalla convergenza dei media, le tecnologie digitali ed una cultura dell'informazione radicalmente trasformata pongono questioni difficili per il fu- 
turo: come facciamo a finanziare i media ed i giornalisti che operano nel pubblico interesse se i modelli di mercato tradizionali sono danneggiati in modo irreparabile? Come facciamo a mantenere l'etica, una particolare precisione ed un dibattito rispettoso quando l'utenza di internet, frammentata e autoreferenziale, rappresenta una parte crescente del processo di ricerca e di diffusione di notizie? E qual è il futuro del giornalismo se il sostegno da parte della politica, le pubbliche relazioni e i meri interessi personali stanno diventando sempre più le forze trainanti del settore mediatico?

Nell'ambito di questa ricerca di soluzioni, il presente capitolo analizza la natura e l'importanza del giornalismo etico e la sua collocazione nello spazio informativo dell'Europa moderna. Vedremo quali saranno alcune delle sfide etiche che affrontano i giornalisti ed i caporedattori nel loro lavoro quotidiano.

I giornalisti, i quali agiscono in un settore determinato della libertà di espressione, godono della tutela della libertà di parola, ma in modo illimitato. La Convenzione europea dei diritti dell'uomo, per esempio, mette in chiaro che le restrizioni alla libertà di espressione potrebbero risultare necessarie nell'interesse, per esempio, della sicurezza nazionale e della pubblica sicurezza. In ogni caso, le eccezioni alla regola base, secondo la quale ciascuno gode della libertà di parola, devono essere previste dalla legge, perseguire un interesse legittimo ed essere necessarie in una democrazia. Ciò è di particolare importanza per i giornalisti il cui lavoro consiste nel controllare l'esercizio del potere e per quelli che confidano nel fatto che la tutela del pubblico interesse consenta loro di fare ciò. Qualsiasi forma di censura, le leggi restrittive e altre misure finalizzate al controllo dei media tendono a sortire un effetto "dissuasivo" sui media, ma anche ad avere un impatto negativo sulla società nel suo complesso, incluso l'intero settore dei diritti umani.

Il giornalismo è una forma espressiva non del tutto libera. Infatti, si tratta, come verrà illustrato nel presente saggio, di una forma di espressione limitata, all'interno della quale la comunità mediatica stessa sviluppa concetti etici che servono a tutelare interessi individuali o di gruppo da forme di abuso inaccettabili. Questa restrizione volontaria contraddistingue il giornalismo dal variegato mondo della libertà di espressione e mostra, allo stesso tempo, che gli interventi dello Stato volti a regolare o controllare il settore non sono necessari o auspicabili.

Nel presente saggio, per "giornalismo etico" si intende il modo in cui i reporter, i redattori e gli altri soggetti coinvolti forniscono, ricercano, preparano e diffondono notizie e commenti sugli eventi che influenzano le vite delle persone. Esso affonda le proprie radici nei valori morali e si è svi- 
luppato parallelamente alla protezione dei diritti umani in Europa. In sostanza, il giornalismo etico serve a soddisfare il diritto all'informazione del pubblico. Si tratta di professionisti anche in quanto ricercano la verità e ne evitano le alterazioni. Si tratta, nel complesso, di concetti etici da promuovere.

Questi principi base forniscono un punto di partenza utile. Possono creare affiatamento nelle redazioni e sono importanti per consolidare la fiducia del pubblico nel valore delle informazioni e delle notizie indipendenti. Esistono, tuttavia, molte altre sfide, incluse le politiche per rafforzare i finanziamenti pubblici al giornalismo incentrato sugli interessi pubblici e per investire in misure finalizzate alla creazione dell'affiatamento per promuovere il pluralismo e la diversità.

Il mondo dell'informazione sta attraversando un processo di trasformazione radicale, ma la necessità di un maggiore accesso pubblico ad informazioni affidabili, utili e puntuali come contraltare alle voci, alle speculazioni ed alla propaganda rimane costante. Per raggiungere tutto ciò, è richiesto un forte impegno per rafforzare il giornalismo etico e l'aumento dell'accesso, da parte del pubblico, a media liberi e indipendenti. Si tratta di parti essenziali della struttura della democrazia e, affinché possiamo difenderle, dobbiamo comprendere meglio in che modo sono definite le informazioni nella sfera pubblica europea nell'era digitale.

\section{LA DIFFERENZA TRA IL GIORNALISMO E LA LIBERTÀ DI ESPRESSIONE}

Come precedentemente affermato, il giornalismo etico si è sviluppato nei decenni scorsi parallelamente allo sviluppo dei diritti umani e della democrazia in Europa, ma oggigiorno il giornalismo ed i diritti umani si intersecano in un momento di grande cambiamento storico collegato alla globalizzazione ed all'esplosione dei media digitali.

In questo contesto, è utile cominciare da un'analisi dell'esigenza di un nuovo dibattito sulle sfide etiche che il giornalismo e la libertà di espressione si trovano ad affrontare.

Il giornalismo copre una comunità informativa molto più ampia della famiglia tradizionale composta dalle persone definite professionisti dei media. Siamo, infatti, di fronte ad un fenomeno in grado di comprendere chiunque si impegni nella diffusione di informazioni per un pubblico interesse. Il giornalismo aggiunge alla libertà di espressione una serie di standard etici e professionali. Al giornalismo è affidato il compito pubblico, da 
svolgere in maniera quanto più onesta ed indipendente possibile, di fornire alla comunità informazioni accurate ed affidabili.

Permane, comunque, uno stretto legame tra l'etica del giornalismo e gli standard dei diritti umani e, in particolare, la libertà di espressione. I giornalisti nutrono, così come i governi, un legittimo interesse alla difesa ed alla promozione di standard elevati nell'ambito di diritti umani e di un giornalismo indipendente.

Ma anche i nuovi attori nutrono degli interessi. Persone esterne alla redazione, le quali aiutano a ricercare, preparare e diffondere le informazioni - si tratta di blogger o altri divulgatori non tradizionali - costituiscono una parte fondamentale del nuovo ambiente creato dai media digitali e da nuove forme di comunicazione note con l'espressione "giornalismo aperto e collegato". Anch'essi svolgono un ruolo importante nella creazione di una nuova cultura di comunicazioni responsabile che può essere coltivata e sostenuta rispettando gli standard dei diritti umani.

Allo stesso tempo, il giornalismo si trova ad affrontare, essendo un bene pubblico, varie pressioni e, in molti Paesi europei, l'indipendenza dei media pubblici esistenti non è garantita. Ciò rappresenta una sfida che richiede un processo di riflessione ed azioni da parte dei giornalisti, dello Stato e della società civile.

Il giornalismo etico è sempre stato nel fulcro delle concezioni di professionalità dei media ed è sempre stato tenuto in considerazione nella creazione di codici deontologici per i giornalisti e di forme di auto-regolamentazione, sia a livello locale, sia a livello nazionale.

Il dibattito sul comportamento etico non è accademico o teorico, bensì dovrebbe essere radicato nelle realtà sociali e politiche del lavoro giornalistico. Se i codici riflettono le aspettative di responsabilità dei giornalisti, essi devono anche essere implementati in un contesto pratico per mezzo di linee guida dettagliate e momenti di formazione sviluppati dai professionisti dei media stessi. Inoltre, l'auto-regolamentazione dei media viene ritenuta un mezzo apprezzabile di risoluzione dei conflitti, di protezione dell'indipendenza del giornalismo, di promozione di standard etici e di riduzione dei rischi di conseguenze giuridiche per i giornalisti.

Vi sono, ad ogni modo, molte iniziative finalizzate alla promozione attiva degli standard etici del giornalismo e della protezione dei diritti all'informazione a livello internazionale, europeo e nazionale. Questi casi possono fungere da esempi per una buona pratica futura.

Bisogna, tuttavia, fare molto di più per rafforzare l'attaccamento ai valori etici dei giornalisti e di altri soggetti che tentano di fornire informazioni tramite comunicazioni pubbliche responsabili. Per fare ciò, è necessario analizzare cosa fa il giornalismo ed il lavoro di persone che operano in set- 
tori comunicativi di pubblico interesse tradizionali e non in maniera distinta dalla libertà di espressione.

Nonostante il diritto alla libertà di espressione riguardi, come formulato nell'articolo 10 della Convenzione Europea per i Diritti dell'Uomo, una moltitudine di forme di espressione, i giornalisti collocano il loro ruolo nell'ambito della libertà di stampa, la quale è una forma di espressione che sostiene la ricerca della verità.

L'importanza chiave della libertà di stampa consiste nel fatto che essa è inserita in un discorso in cui le varie opinioni non vengono solo espresse, ma anche messe alla prova sotto forma di dibattito aperto. La libertà di espressione nel senso più lato non sostiene la ricerca della verità. Conferisce a chiunque il diritto di esprimersi, entro determinati limiti normativi chiaramente delineati, su tutto quanto, come e quando vuole. Le opinioni hanno il diritto di essere decenti o indecenti, oneste o disoneste, eque o faziose.

Oltre dieci anni fa, Onora O'Neill, professoressa di filosofia presso l'Università di Cambridge, ha definito "autoreferenziale" la libertà di espressione illimitata, in contrapposizione all'aspirazione "altruista" del giornalismo e dei media, i quali sono guidati dagli ideali chiave della loro missione ed ambiscono a determinati standard e valori (O'Neill 2004).

La distinzione effettuata dalla prof.ssa O'Neill è diventata particolarmente importante nell'era dei social network e delle comunicazioni digitali. Oggi, il mondo di Twitter, Facebook e delle chat online crea, nel complesso, una pletora di chiacchiere autoreferenziali e sfrenate, un dibattito e, talvolta, forme di comunicazione segnati dalla violenza. Il giornalismo non è, di conseguenza, la libertà di espressione. Si tratta di un'espressione regolamentata, segnata dai principi etici della mission giornalistica - affermare la verità, indipendenza, responsabilità, equità e rispetto del prossimo. Si tratta di un accordo verso una regolamentazione volontaria che solitamente non si trova sul panorama delle informazioni moderne aperte e che, infatti, non verrebbe riconosciuto da parte di molti blogger e utenti dei social network, i quali insistono con fermezza sul loro diritto alla libertà di espressione.

$\mathrm{Ma}$, affinché i giornalisti siano in grado di esercitare questa forma di auto-censura, devono essere in grado di lavorare liberi da pressioni ed intimidazioni. Nell'ampio mondo delle pubbliche comunicazioni, nel quale i giornalisti lavorano di pari passo con il pubblico, una parte del quale fornisce altresì il lavoro giornalistico, l'esigenza di creare un ambiente sicuro, libero da pressioni è essenziale. Per questi motivi, i giornalisti nutrono, così come i governi, un interesse legittimo alla difesa ed alla promozione di elevati standard in materia di diritti umani. 


\section{DALLA CRISI A UNA NUOVA CONCEZIONE DEL GIORNALISMO ETICO}

Gli spettacolari cambiamenti degli ultimi vent'anni introdotti da internet e dalle comunicazioni digitali hanno rivoluzionato il mondo dei media, ma non è possibile spostare indietro le lancette dell'orologio.

La seguente considerazione del Presidente degli Stati Uniti d'America Barack Obama del luglio del 2013 (Blum 2013) riassume la realtà che affrontano i giornalisti tradizionali ed i media in Europa:

Una volta lì esistevano giornali locali dappertutto. Se volevi fare il giornalista, potevi vivere bene lavorando per il quotidiano della tua città. Ora hai una manciata di giornali che fanno guadagni perché si tratta di marchi nazionali, ed i giornalisti devono lottare per guadagnarsi un'esistenza, lavorando in certi casi come freelancer e senza i medesimi benefici di cui godrebbero se avessero un impiego regolare presso un giornale. Ciò che vale per il giornalismo vale per il settore manifatturiero e per il commercio. Dobbiamo ammettere che quei vecchi tempi non torneranno.

In ogni caso, in un clima in cui il cambiamento radicale minaccia di schiacciare i valori etici dei media tradizionali, deve cambiare qualcosa per riaffermare tali valori. Il processo di cambiamento, nonostante sia stimolante e liberatorio per molti utenti e per molte persone nell'industria della comunicazione, non ha generato un grande sentimento di ottimismo nelle redazioni d'Europa. Infatti, vi è una perdita di fiducia ed in molte parti del giornalismo e del settore mediatico la morale è bassa. Il giornalismo a servizio del pubblico interesse è messo a repentaglio dalle condizioni sociali e professionali incerte e precarie in cui si trova a lavorare, dalla mancanza di regolamentazioni e dai timori crescenti nei confronti di una maggiore influenza dello Stato, di un'intromissione politica e di contenuti dettati da interessi aziendali.

Il riassetto del settore mediatico è stato guidato da convergenze tecnologiche e da un cambiamento rivoluzionario nel modo in cui le persone comunicano e diffondono informazioni. Quando internet ha aperto nuovi mercati, i media tradizionali hanno assistito al collasso dei loro modelli commerciali. Le entrate pubblicitarie sono sprofondate e le previsioni sugli introiti sono disperate. Anche i media digitali più seguiti lottano per rimanere concorrenziali da quando i giganti di internet quali Google, Amazon e Facebook sottraggono loro gli introiti pubblicitari.

La crisi economica che la maggior parte dei media si trova ad affrontare ha comportato una riduzione delle risorse disponibili per mantenere e raf- 
forzare il lavoro giornalistico. In sostanza, si spende di meno nell'impiego e nella formazione e rimane a disposizione meno tempo per la ricerca e la valutazione dei fatti, rafforzando, di conseguenza, lo scetticismo pubblico e la minore fiducia. Alcuni media sono divenuti social network e fonti digitali imprevedibili ed inaffidabili al fine di colmare i vuoti pubblicitari ed editoriali. Promuovono commenti on-line per incoraggiare la partecipazione del pubblico nella speranza di ottenere potenziali pubblicità automatizzate lucrative. Utilizzano contenuti generati dagli utenti per soddisfare i voraci appetiti della macchina informativa in funzione 24 ore al giorno e 7 giorni alla settimana. Nel frattempo, altri giornalisti e media, i quali rifuggono la politica che antepone il numero di click ai contenuti, dirigono lo sguardo verso fondazioni private, filantropie o nuovi modelli di sostegno pubblico per sorreggere l'integrità del giornalismo e per fare ciò senza compromettere l'indipendenza editoriale.

Lo strumento utilizzato per tutelare il giornalismo ed il lavoro editoriale dalla pubblicità è collassato. Le pubblicità redazionali, le rubriche sponsorizzate ed editoriali collegati ad interessi economici si trovano di frequente. I vecchi difetti sono stati amplificati dalle nuove tecnologie. La tradizionale ossessione dei giornali tabloid per il sesso, la violenza ed il sensazionalismo è stata superata da internet e dai social media. Una "gara per la pubblicazione" diffonde pettegolezzi, speculazioni e forme di ignoranza in modo virale.

La disinformazione e gli stereotipi prendono di mira i gruppi più vulnerabili e marginalizzati e rinvigoriscono l'odio ed i pregiudizi. La propaganda politica e le pubbliche relazioni sono risucchiate nel vuoto quando il giornalismo ben ricercato viene tranciato, in un processo noto con il termine "churnalism" (Davies 2008). Una competizione mediatica estrema, un mercato sempre più debole per un giornalismo di qualità e l'influenza crescente dei social network che dettano l'agenda mediatica comportano dei costi.

Non sorprende che in questo ambiente molti media non siano in grado di soddisfare le loro aspirazioni di onesti e accurati narratori della verità. I giornalisti ed i caporedattori affrontano ostacoli che impediscono loro di fornire informazioni all'altezza delle loro ambizioni etiche. I giornalisti hanno sempre dovuto affrontare le intromissioni politiche ed aziendali, ma oggigiorno si tratta di intromissioni segrete, pervasive ed insidiose, e vi sono stati tagli negli investimenti editoriali che mettono a repentaglio il tessuto giornalistico. Il giornalismo investigativo si fa più raro e le voci delle comunità marginalizzate e vulnerabili si riducono. Il giornalismo non è visto di buon occhio in quanto i pettegolezzi, le speculazioni e le banalità 
sostituiscono un giornalismo che fornisca ciò che le persone hanno diritto di sapere e che alimenti la società civile.

Ma, nel mezzo di queste turbolenze, esiste dell'ottimismo. Molti giornalisti e professionisti dei media hanno iniziato a respingere la scarsa morale causata dalle crescenti pressioni governative e dalla natura ingiusta e precaria del loro lavoro. Sono sempre più numerosi gli appelli all'interno dei media che richiedono, per il futuro, una visione nuova e rinvigorita del giornalismo, basata su una ripresa dello spirito della mission e dei valori e da una nuova e inclusiva partnership mediatica con il pubblico.

Molti ritengono che il giornalismo possa fungere da guida per un tipo di comunicazioni responsabile all'interno della sfera delle informazioni in Europa in grado di essere una fonte di ispirazione per altri, ma ciò si verificherà soltanto se i gruppi di operatori professionisti del settore mediatico collaboreranno per rafforzare il mestiere del giornalismo e per costruire la fiducia del pubblico nei media.

Fu questo concetto di costruzione della solidarietà in una comunità mediatica divisa a portare al lancio di Ethical Journalism Network nel 2012, ${ }^{1}$ un'associazione di editori, caporedattori e operatori mediatici di tutte le piattaforme dei media, i quali richiamavano alla cooperazione tra i media, a gruppi di supporto reciproco tra i giornalisti, ad un rifiuto, da parte del pubblico, di interferenze governative nel lavoro dei media ed alla collaborazione in favore dell'etica, della buona pratica e dell'auto-regolamentazione in difesa del giornalismo di qualità.

Questa iniziativa risponde a due tendenze importanti, le quali hanno entrambe creato disagi ai giornalisti, ma, allo stesso tempo, fornito opportunità per migliorare il difficile clima in cui i media operano.

La prima rappresenta lo smantellamento della struttura elitaria e professionalizzata con la quale i media tradizionali hanno detenuto un potere enorme nel controllare l'agenda mediatica e nel formare l'opinione pubblica. Oggigiorno, i media collaborano strettamente con il loro pubblico, il quale può, grazie alla tecnologia, rispondere immediatamente a quello che vede, sente e legge. Le opportunità per coinvolgere il pubblico nella preparazione e nella diffusione di notizie ed informazioni costituiscono opportunità per ampliare l'ambito di copertura giornalistica, per rendere i media ed il giornalismo più responsabili e per rafforzare il dibattito sugli standard da osservare al di là delle redazioni nelle battaglie per migliorare la qualità dei contenuti nelle comunicazioni quotidiane.

1 Vedi http://ethicaljournalismnetwork.org/en, consultato il 16 luglio 2015. 
Il secondo cambiamento consiste nell'utilizzo della tecnologia per riorganizzare e rimettere a fuoco il lavoro del giornalismo al fine di renderlo più efficace nell'ottemperare il proprio compito di agire da "guardiano" e nel fornire al pubblico informazioni rilevanti, utili e precise.

L'uso liberatorio della tecnologia, della stessa tecnologia di cui abusano i governi per spiare i lori cittadini o i giornalisti o che viene sfruttata da altri per diffondere messaggi d'odio, può anche essere un bene. Può essere utilizzata per aumentare la trasparenza, aumentare gli standard e costruire fiducia nella democrazia, e non soltanto all'interno dei circoli mediatici tradizionali.

\section{I PRINCIPI ETICI FONDAMENTALI DEL GIORNALISMO}

Per trarre vantaggio da questo cambiamento di circostanze, è importante comprendere meglio le fondamenta etiche del giornalismo e ciò che lo distingue dal mero esercizio della libertà di espressione, nonché valutare come questi valori possano essere utili nella promozione di un tipo di comunicazioni pubbliche civili e responsabili all'interno della comunità in generale.

Nonostante, nella maggior parte dei Paesi, non tutti gli operatori mediatici conoscano perfettamente a memoria i codici sviluppati per favorire un giornalismo etico, la maggior parte degli editori, caporedattori e del personale di redazione è consapevole delle proprie responsabilità e delle finalità del giornalismo.

I principi etici del giornalismo vennero elaborati per la prima volta in Gran Bretagna negli anni 50 dell'Ottocento ed i primi codici per il giornalismo risalgono agli anni 30 del secolo scorso. Oggigiorno esistono più di 400 codici e dichiarazioni in tutto il mondo. ${ }^{2}$ Derivano da un'ampia varietà di tradizioni culturali ma, fondamentalmente, tutti fanno riferimento ai medesimi cinque valori chiave che consistono nell'imposizione volontaria di regole e demarcano l'ambito della libertà di espressione in cui opera il giornalismo professionale.

2 Una delle raccolte più ampie di codici è quella redatta da by Jean-Claude Bertrand, la quale viene ora aggiornata da Ethical Journalism Network e dalla Missouri School of Journalism. Vedi www.rjionline.org/media-accountability-systems, consultato il 16 luglio 2015. 
Si tratta dei seguenti valori:

\section{Precisione e comunicazioni basate sui fatti}

I giornalisti non possono sempre garantire la "verità", ma comprendere bene i fatti rappresenta un principio cardine del giornalismo. I giornalisti dovrebbero sempre battersi per la precisione, fornire tutti i fatti rilevanti di cui sono a conoscenza e assicurare che siano stati appurati. Quando non sono in grado di valutare l'esattezza delle informazioni, devono renderlo noto.

\section{Indipendenza}

I giornalisti devono essere voci indipendenti; non dovrebbero agire, in modo formale o informale, per conto di interessi particolari di natura politica, commerciale o culturale. Dovrebbero essere trasparenti nei confronti dei loro titolari e del loro lavoro. Dovrebbero dichiarare ai caporedattori - o al loro pubblico - qualsiasi affiliazione politica, eventuali legami economici o altre informazioni personali che potrebbero dare luogo ad un conflitto di interessi.

\section{Correttezza e imparzialità}

Molte storie hanno almeno due lati. Mentre non vi è alcun obbligo di presentare qualsiasi aspetto in ogni articolo, le storie giornalistiche dovrebbero essere bilanciate e contestualizzate. L'oggettività non è sempre possibile, e forse non sempre auspicabile (in casi di, per esempio, brutalità o di disumanità), ma una descrizione imparziale crea fiducia e confidenza.

\section{Umanità}

I giornalisti non dovrebbero nuocere. Ciò che pubblicano o trasmettono potrebbe essere offensivo. Dovrebbero essere consapevoli dell'impatto delle parole e delle immagini sulle vite degli altri, in particolare sui gruppi vulnerabili, sulle comunità marginalizzate e sulle minoranze. Dovrebbero essere particolarmente sensibili al pericolo di contribuire all'incitamento all'odio o alla violenza.

\section{Responsabilità}

Un chiaro segno di professionalità e di giornalismo responsabile consiste nella capacità di essere responsabili e trasparenti. Quando i giornalisti commettono un errore, devono correggerlo, e le scuse devono essere rese in maniera sincera e non cinica. I giornalisti ed i media devono prestare ascolto alle preoccupazioni del pubblico. Essi non cambieran- 
no ciò che i lettori scrivono o dicono, ma devono fornire rimedi a giudizi scorretti.

Questa cornice etica rappresenta un punto di partenza e molti media hanno sviluppato linee guida redazionali complete per aiutare i giornalisti, gli autori ed i caporedattori ad affrontare la moltitudine di dilemmi che sorgono nel lavoro quotidiano. La consapevolezza dell'importanza dell'etica nel giornalismo ha portato ad un numero crescente di datori di lavoro che includono i codici redazionali nei contratti di lavoro, così come ad un crescente interesse nei confronti di nomine di difensori redazionali e dei c.d. readers' editors.

\section{DEFINIRE GLI ATTI DI GIORNALISMO E CHI È UN GIORNALISTA}

Questi sviluppi rappresentano segni incoraggianti che si sostanziano in una tendenza verso una visione rinnovata per il giornalismo in Europa, formata anche dai gruppi mediatici professionisti.

Le organizzazioni giornalistiche, per esempio quelle che fanno parte della Federazione europea dei giornalisti, così come quelle dei datori di lavoro del settore mediatico, quale l'Associazione mondiale della carta stampata, stanno intraprendendo alcune iniziative per costruire un maggiore sostegno pubblico al giornalismo che opera nell'interesse pubblico. Stanno altresì cercando una nuova solidarietà in un settore diviso, una risposta positiva alle sfide che comporta la rivoluzione dell'informazione e, soprattutto, un futuro sicuro ed etico per il mestiere del giornalismo. L'obiettivo è semplice: consolidare i valori dell'onesta, della verità e delle finalità pubbliche nei media e costruire la fiducia nel giornalismo come strumento di espressione democratica, come garanzia di libertà di parola, come interprete affidabile e fidato del mondo complesso in cui viviamo.

Ma chi è il giornalista e chi si assumerà tale responsabilità? Nell'ambiente mediatico di oggi vi è un ampio dibattito su chi sia un giornalista e chi possa, di conseguenza, godere delle protezioni che andrebbero garantite ai soggetti che svolgono il lavoro giornalistico, quale la protezione delle fonti. Si tratta di una questione importante e per nulla accademica. Lo Stato di diritto richiede in quest'ambito - per esempio nella garanzia di protezione delle fonti giornalistiche dell'informazione, o nel prendere in considerazione l'interesse pubblico nei casi di diffamazione - che i legislatori ed i giudici abbiano idee chiare in merito alle circostanze in cui applicare tali protezioni e su chi ne debba godere. 
I giornalisti sono stati tradizionalmente definiti tramite il loro rapporto di lavoro presso un'azienda del settore mediatico, tramite l'appartenenza ad un'associazione di categoria o ad un sindacato o tramite il completamento di un determinato corso di studi. Il giornalismo è un mestiere dotato di una duplice personalità. Il professionista esperto e creativo a tempo pieno è parte di un gruppo mediatico, lavora in una squadra volta a una missione definita dal proprietario o dalla dirigenza. Al contrario, il giornalista freelance, a tempo parziale o a contratto, vende i propri servizi, spesso in condizioni precarie, e questi ultimi sono diventati la categoria in maggiore ascesa della professione. Il termine "giornalista" viene utilizzato sempre di più per includere tutti coloro che regolarmente raccolgono o diffondono informazioni al pubblico perseguendo scopi giornalistici, per esempio i blogger. Anche coloro che non hanno completato un percorso formativo, che non sono iscritti a un'associazione di categoria e che non hanno un impiego presso i media possono commettere quelli che potrebbero essere definiti "atti di giornalismo".

Molti potrebbero ora ritenere che chiunque pubblichi informazioni su questioni di pubblico interesse dovrebbe, in questo contesto, beneficiare della protezione e dei privilegi giuridici limitati accordati ai giornalisti a tempo pieno, inclusa la protezione dalla censura e da interferenze indebite, il diritto di pubblicare in sicurezza ed il diritto di proteggere la confidenzialità delle fonti.

Considerando la questione della protezione, il problema dell'intenzione di pubblicare nel pubblico interesse o l'adesione a e la consapevolezza di determinati valori etici, per esempio quelli precedentemente elencati, diventerà sempre più importante per assicurare che la protezione legittima sia accordata a chiunque (inclusi i giornalisti professionisti e non) ne abbia diritto. Coloro che ambiscono a comunicare nel pubblico interesse dovrebbero riconoscere l'esigenza di rivelare qualsiasi affiliazione o appartenenza rilevanti. Solo gli atti di giornalismo compiuti in buona fede sono degni di protezione.

Fortunatamente, il giornalismo non è più l'élite autoreferenziale di una volta. Ma una concezione più ampia di coloro che praticano il giornalismo non giustifica un indebolimento delle capacità, degli standard o dei valori etici e non dovrebbe neanche ridurre in nessun modo le richieste legittime di coloro che operano all'interno del giornalismo e che richiedono un sostegno maggiore nella difesa del proprio lavoro e un miglioramento delle condizioni di lavoro.

Nonostante il giornalismo sia diventato una professione più aperta, coloro che ne vivono sono diventati le vittime più celebri della crisi economica in Europa così come in tutto il mondo. I posti di lavoro sono diventati 
precari e le condizioni di lavoro sono peggiorate. I giovani che cercano di trovare un appoggio nel giornalismo subiscono l'umiliazione di lunghi tirocini non pagati in quanto competono tra di loro per le poche occasioni di carriera disponibili. I sindacati e le associazioni di categoria condannano giustamente il calo di investimenti nei posti di lavori, nella formazione, nelle condizioni sociali e nella capacità professionale. Avvertono che indebolire la condizione del lavoro giornalistico mette a repentaglio la democrazia.

Creare nuove strutture per la cooperazione e puntare sulla qualità può stimolare la nuova generazione di giornalisti, che è più giovane, più variegata e non meno legata ai valori del personale redazionale tradizionale, ad aderire alla crescente opinione del settore per migliorare e rafforzare il giornalismo ed i contenuti mediatici. Creare questo nuovo ampio movimento non è un compito facile date le sfide etiche emergenti.

\section{LA SFIDA ETICA: COSTRUIRE FIDUCIA NELL'AUTOREGOLAMENTAZIONE}

L'autoregolamentazione comincia con il singolo, e si consolida nelle redazioni e nelle case mediatiche, ma, affinché si possa incidere nella coscienza pubblica, è altresì necessario lavorare in modo efficiente nell'industria dei media a livello nazionale.

Il principio di regolamentazione volontaria dei media, ove i giornalisti lavorano in collaborazione con rappresentanti del pubblico, è il modo migliore per costruire un rapporto di fiducia nei media. Ma nonostante la teoria dell'autoregolamentazione dei media venga compresa sempre meglio, vi sono molte questioni aperte: ci si chiede se tali sistemi, in particolare quelli che riguardano la stampa, ne siano in grado in un'epoca di crescenti pressioni politiche e commerciali sul giornalismo.

I recenti scandali di hackeraggio di telefoni cellulari e di corruzione della stampa nel Regno Unito che hanno coinvolto il gigante mondiale dei media News Corporation, di proprietà di Rupert Murdoch, che ha portato alla chiusura di un importante quotidiano e all'incarcerazione di alcuni giornalisti, forniscono una prova scioccante dell'incapacità di autoregolamentarsi a livello individuale, aziendale e settoriale. ${ }^{3}$

3 Per una relazione completa sugli scandali e la conseguente inchiesta Leveson vedi: http://webarchive. nationalarchives.gov.uk/20140122145147/http:/www.levesoninq uiry.org.uk/, consultato il 16 luglio 2015. 
Una vittima di alto rango fu la Press Complaints Commission, un regolatore di settore di fama mondiale, il quale venne ritenuto del tutto inefficace nel tenere a freno gli eccessi del giornalismo tabloid e incapace di fornire forme di risarcimento adeguate ed accettabili alle vittime degli abusi di stampa. Venne chiusa nel 2014. Nel Regno Unito sono state adottate alcune riforme che hanno visto la creazione di una nuova organizzazione, la Independent Press Standards Organisation, tuttavia rimangono aperte alcune serie questioni sulla sufficiente indipendenza di questa nuova organizzazione dall'industria mediatica e sulla credibilità della sua autoregolamentazione. Tre delle maggiori testate, The Guardian, Financial Times e The Independent, si sono rifiutate di aderirvi.

Per costruire la fiducia del pubblico il giornalismo deve mettersi in rete con il proprio pubblico e assumersi la responsabilità dei propri errori. I giornalisti dovrebbero vedere un'autoregolamentazione indipendente come un modo di rafforzare il loro operato. I sistemi di responsabilità dei media credibili costituiscono il fulcro della creazione della fiducia del pubblico nei media e, senza di essi, le persone non dispongono di alcuna forma di effettiva valutazione dei media o possibilità di sfidare gli abusi del potere quando il giornalismo deraglia.

La maggior parte dei media sarebbe d'accordo, tuttavia molti giornalisti e molte organizzazioni mediatiche sono restii ad aprirsi. Molti hanno fatto cattive esperienze con la maniera intrusiva in cui i governi utilizzeranno gli argomenti di regolazione come scusa per monitorare ed interferire nel modo in cui i media lavorano. Ciò avvenne sicuramente in Ungheria nel 2011, quando venne approvata una nuova legge sui media che introdusse un organo di controllo sui media i cui membri vennero nominati dal partito di governo. Introdotta sullo sfondo di proteste per una mancata regolamentazione dei media, la legge causò un'ondata di proteste nel resto d'Europa (Human Rights Watch 2011). ${ }^{4}$

Ciononostante, il fatto che i giornalisti siano notoriamente restii ad ammettere i propri errori è una verità ben nota e accettata all'interno ed all'esterno dei media. Questa mancanza di umiltà è bizzarra se pensiamo a come i media possano rivelarsi spietati nella critica di molte persone della vita pubblica.

Questa invincibile riluttanza nell'accettare il concetto di regolamentazione indipendente e nell'accettare riforme importanti sul modo in cui i media affrontano le loro questioni interne, in particolare nella stampa,

4 La controversia sulla nuova nuova legge sui media ha sollevato una serie questioni sui diritti umani. Vedi Human Rights Watch (2011). 
comporta una crisi di fiducia, come lo si è visto nel Regno Unito in seguito agli scandali di hackeraggio dei cellulari degli ultimi anni.

Si tratta, in sostanza, di una forma di autodifesa. Le lamentele ignorate o prese poco sul serio possono creare danni enormi, mentre spesso un veloce diritto di replica o la pubblicazione di una rettifica ristabiliscono la convinzione che il giornalismo vada difeso. I professionisti dei media, a loro volta membri del pubblico, possono aiutare ad influenzare il dibattito su come rendere i media più credibili e affidabili.

L'interesse delle organizzazioni di stampa a proteggere loro stessi da interferenze esterne è comprensibile, ma quando comporta un indebolimento della legittima valutazione pubblica del loro lavoro ciò può rappresentare un ostacolo ad un'effettiva autoregolamentazione. Allo stesso tempo, la convergenze di tecnologie della comunicazione, per esempio, ha reso obsoleta la tradizionale distinzione tra i media audiovisivi e la carta stampata. $\mathrm{Ci}$ si chiede, quindi, per quale motivo esistano ancor oggi, nella maggior parte dei Paesi, strutture e normative differenti per la trattazione di pubbliche rimostranze sui contenuti del giornalismo nella stampa ed in televisione.

In alcuni Paesi, per esempio in Norvegia, nei Paesi Bassi e nel Belgio, tutto il giornalismo pubblicato su qualsiasi piattaforma viene giudicato da un singolo consiglio di stampa o mediatico. Questi modelli andrebbero presi ad esempio? Il compito di un organo di autoregolamentazione nazionale dovrebbe derivare da un codice comportamentale contenente la pratica del giornalismo, il quale andrebbe condiviso dalla comunità dei professionisti dei media.

Vi sono alcuni principi base per l'autoregolamentazione a livello statale:

- L'organo di autoregolamentazione del giornalismo deve agire in maniera indipendente dal governo e dal parlamento.

- Dovrebbe creare un equilibrio nell'iscrizione e nella rappresentanza tra i giornalisti di tutte le piattaforme mediatiche ed il pubblico, rappresentando un'ampia fetta della società civile.

- L'iscrizione all'organo autoregolatore indipendente dovrebbe essere decisa da un gruppo di cariche in forma indipendente dal governo e dall'industria mediatica, la quale dovrebbe essere a sua volta designata in modo indipendente.

- Il processo di regolamentazione dei contenuti dovrebbe includere, a tutti i livelli, le persone che attivamente si impegnano nel giornalismo e persone che comprendono come funzionano il giornalismo ed i media. 
- Laddove esiste una definizione statuale di tale processo di autoregolamentazione, esso dovrebbe essere definito esattamente e limitato alle procedure di validazione, e non prevedere alcuna interferenza giuridica nelle attività operative di un regolatore indipendente.

- Qualsiasi statuto deve prevedere un obbligo esplicito per il governo e le autorità dello Stato di sostenere e proteggere la libertà di stampa.

Non esiste un modello universale, ma vi sono molti esempi di buona pratica, laddove il compito viene svolto in maniera effettiva e competente da parte di autorità che hanno ottenuto ampio consenso e detengono la fiducia del pubblico. In Norvegia, Danimarca, Germania ed in molti altri Paesi europei i comitati nazionali della stampa hanno ampio successo perché i proprietari, i giornalisti ed $\mathrm{i}$ caporedattori hanno acconsentito alla collaborazione per svolgere un lavoro di autoregolamentazione, dal punto di vista sia pratico che teorico.

E come facciamo a finanziare i sistemi di responsabilità se i modelli di finanziamento tradizionali che fanno affidamento alle disponibilità economiche del settore stanno collassando?

In molti Paesi, i mercati tradizionali dei media si trovano in caduta libera, le tirature sono basse e le inserzioni pubblicitarie calano a favore di opportunità on-line maggiormente lucrative. Dappertutto si denota una crescente consapevolezza della necessità di maggiori contributi pubblici per i sistemi di responsabilità dei media. Vi è una forte argomentazione secondo la quale sussiste, in fondo, un sano interesse pubblico nell'avere un sistema giornalistico credibile, responsabile ed etico. In ogni caso, utilizzare i fondi dei contribuenti per la responsabilità dei media richiede particolare attenzione. I fondi pubblici non devono compromettere i principi della regolamentazione indipendente.

Una questione chiave riguarda ciò che succede quando un giornale o un'organizzazione mediatica si sottraggono ad un consiglio di stampa e si rifiutano di pubblicare una rettifica o di fornire un ristorno quando violano i principi etici. L'opinione diffusa dei media si esprime contro forme di coercizione giuridica, preferendo i sistemi su base volontaria e la pressione reciproca. In ogni caso, deve esserci un meccanismo per affrontare le offese ingiuriose che superano una determinata linea rossa.

In Svezia, il consiglio della stampa sanziona i media che violano le regole e, nonostante si tratti di un sistema su base volontaria, chiunque paga in quanto le aziende del settore nutrono fiducia nel procedimento. In Austria il sistema è collassato quando un quotidiano ha apertamente sfidato una decisione del consiglio della stampa. Di conseguenza, il consiglio della stampa si è autoleso. Ci sono voluti anni per ristabilire la fiducia delle 
aziende del settore per rimettere in carreggiata la stampa. La coercizione normativa è prevista in Danimarca, ma il suo campo di applicazione è definito con precisione e tale strumento viene applicato soltanto in circostanze eccezionali. Non si tratta di un modello che molti vedono con favore, in quanto può favorire interferenze giuridiche e politiche, ma qualsiasi forma di regolamentazione indipendente dovrebbe essere oggetto di dibattito.

Nella maggior parte dei Paesi, costruire fiducia nell'autoregolamentazione rimane una sfida colossale a livello imprenditoriale e aziendale. La realtà sembra far capire che il giornalismo sta entrando in un'era di transizione non soltanto dal punto di vista del modo in cui i giornalisti lavorano e delle loro relazioni con il pubblico, bensì anche nel modo in cui i reporter e i caporedattori rispondono delle loro azioni.

Tradizionalmente, vi sono due modi di regolare il giornalismo a livello nazionale: un sistema su base volontaria per la stampa e controlli giuridici per le radiotelevisioni.

Queste strutture vennero create per il panorama mediatico di ieri e sono sempre più obsolete. I giornalisti digitali di oggi lavorano su video, carta ed audio contemporaneamente. Di conseguenza, avrebbe più senso disporre di un solo regolatore statale che comprenda tutte le piattaforme giornalistiche.

La questione del finanziamento comporta un'altra difficile problematica. Idealmente, i giornalisti ed i media dovrebbero pagare l'iscrizione ai consigli della stampa, ma in questi giorni di magra i media sono ancora in grado di sostenere tale spesa? La risposta è sempre più negativa. Di conseguenza, chi pagherà in futuro? Forse dovremmo pensare ad utilizzare i fondi pubblici. In definitiva, una regolamentazione indipendente dei media è un'attività che si svolge nell'interesse pubblico. Ma se utilizziamo i fondi dei contribuenti, come facciamo a garantire che ciò non comprometterà l'indipendenza editoriale?

Queste problematiche hanno spinto l'Ethical Journalism Network a commissionare un report, The Trust Factor, pubblicato nel febbraio del 2015 , il quale esamina la credibilità dell'autoregolamentazione in una serie di Paesi, tra cui vari Stati europei (White 2015a).

Il report esamina la questione dell'autoregolamentazione a tre livelli:

\section{A livello individuale}

I giornalisti sono, in generale, liberi di agire secondo la propria coscienza? I giornalisti sono consapevoli dei loro obblighi e dei loro doveri etici e professionali? 


\section{A livello dell'industria mediatica}

Le aziende mediatiche dispongono di sistemi credibili per affrontare le lamentale? Vi sono garanti o reader's editors? I media si sono dati regole interne per monitorare i conflitti di interesse politici o commerciali e la proprietà si comporta in modo trasparente?

3. A livello nazionale inclusi $i$ sistemi di settore

I consigli di stampa su base volontaria ed i consigli dei media sono effettivi e vengono rispettati dai giornalisti e dagli editori? Quale ruolo svolge la legge e chi finanzia l'autoregolamentazione?

Le conclusioni hanno evidenziato debolezze a tutti i livelli. Il report rivela che nel mezzo dei cambiamenti rivoluzionari che il giornalismo sta attraversando vi sono la trasformazione della cultura delle pubbliche comunicazione e la sempre più grande necessità di un giornalismo responsabile. Ciononostante, alcune questioni più ampie rimangono aperte in merito al modo in cui sviluppare sistemi di responsabilità in sintonia con questa nuova era dell'informazione.

Il report ha evidenziato che la Norvegia dispone del modello di autoregolamentazione più rilevante. I media norvegesi hanno creato un sistema credibile a causa del loro impegno determinato verso un giornalismo indipendente e la solidarietà tra media. Il sistema di complaint adottato dalla stampa norvegese funziona perché tutti i player collaborano e seguono un solo codice comportamentale riconosciuto e rispettato all'interno del giornalismo e applicato in maniera eguale dai media su tutte le piattaforme.

Il report ha altresì evidenziato che la Norvegia, unico caso dei media auto-regolamentati al mondo, consente l'accesso del pubblico alle udienze in cui si trattano le vertenze. Si tratta di un livello di trasparenza unico e pone dei modelli che altri potrebbero seguire. È un sistema che opera in stridente contrasto con i Paesi ove i media sono soffocati dalle leggi sui media, come in Ungheria, o dove prevalgono gli interessi aziendali, come nel Regno Unito.

Ma gli standard non vanno realizzati solo all'interno delle redazioni. L'etica e l'autoregolamentazione sono di eguale importanza anche nei consigli di amministrazione. I proprietari e i dirigenti dei media non sono esenti dal mettere in pratica gli standard che richiedono ai propri giornalisti. Infatti, è essenziale per la creazione di media responsabili e liberi che vi sia una condivisione di valori, di mission e di standard del giornalismo dall'apice alla radice della piramide mediatica.

Sussiste un legame consolidato tra la qualità dei mass media e la democrazia. Alcune ricerche recenti illustrano l'importanza dell'esistenza di media liberi nel costruire società aperte in cui predomina un clima di fiducia, 
nonostante ciò non ne sia una garanzia (Myers 2012). Le organizzazioni mediatiche devono a loro volta mettere in pratica standard elevati. Per questa ragione, i proprietari dei media devono promuovere la trasparenza e la buona governance all'interno delle case editrici.

In molte parti del mondo ci si preoccupa del fatto che i caporedattori abbiano perso parte della loro influenza sul lavoro dei giornalisti a vantaggio dei proprietari dei media. La leadership morale del management, o la mancanza della stessa, costituiscono un fattore cruciale nel determinare il comportamento dei giornalisti (Lambeth 1992). I caporedattori spesso definiscono "la" morale del lavoro mediatico in base alle preferenze degli editori i cui conflitti di interessi, determinati da stretti legami con partner politici o d'affari, spesso sono la causa di interferenze palesi o velate nelle redazioni. Quando ciò succede, danneggia inevitabilmente la credibilità del giornalismo. L'Ethical Journalism Network promuove la trasparenza nell'editoria e l'adozione di regole internazionali di buona governance per i media. Agisce nella convinzione che gli editori debbano rispettare i modelli di apertura e di comportamento morale che i loro giornalisti mettono in atto nei confronti di terzi nel lavoro quotidiano.

La creazione di un Ethical Media Audit per aiutare le aziende dei media a sviluppare i loro processi interni di autovalutazione si è già rivelato utile nell'aiutare le aziende in Pakistan a sviluppare forme di autoregolamentazione interne più efficaci (Ethical Journalism Network 2015). Il report evidenzia il motivo per cui la creazione di forme di autoregolamentazione a livello aziendale sia forse la cosa più importante. Costruire un rapporto di fiducia con il pubblico dovrebbe essere l'obiettivo di ogni redazione e la crescita del numero di garanti aziendali o readers' editors rappresenta un segnale positivo che indica come sempre più media stiano prendendo sul serio la questione.

La nomina di garanti responsabili della correzione di errori, i quali spieghino al pubblico come funziona il giornalismo, rappresenta la creazione di un accordo verso la professionalità e la responsabilità e un meccanismo utile per costruire la fiducia del pubblico. ${ }^{5}$

Allo stesso tempo, i giornalisti devono essere informati circa i loro doveri etici e devono disporre di sistemi interni che monitorano costantemente il lavoro delle redazioni, fornendo loro linee guida sullo stile e sulla sostanza del giornalismo, che illustrino i possibili dilemmi etici e come risolvere questi ultimi.

5 Vedi Organization of News Ombudsmen http://newsombudsmen.org/, consultato il 16 luglio 2015. 
Tuttò ciò è più facile a dirsi che a farsi in un'epoca in cui, a fronte dei tagli all'editoria, alcune dirigenze richiedono ancora fondi per riparare agli errori delle redazioni. Secondo una forte argomentazione, mantenere l'onestà nel giornalismo è un buon motivo di spesa per i media e per il pubblico in senso più ampio, e rappresenta un buon investimento per la democrazia, ma molti media e molti politici non condividono tale opinione.

\section{UNA SFIDA ETICA: I CONFLITTI DI INTERESSE ALL'INTERNO DEI MEDIA}

Nonostante le persone del settore mediatico parlino, dall'apice fino al basso, di "missioni", del pubblico interesse e del ruolo cruciale di guardiano degli abusi di potere della nostra élite poltica ed economica che il giornalismo ricopre, i media sono molto meno franchi quando si parla dei disordini che contornano i loro affari.

Come precedentemente affermato, molti media dimenticano la loro ambizione di essere onesti e accurati narratori della verità. I giornalisti ed i caporedattori affrontano, tuttavia, anche ostacoli interni che impediscono loro di fornire notizie sulla base delle loro alte ambizioni etiche. I giornalisti hanno sempre dovuto affrontare le intromissioni politiche e aziendali, ma oggigiorno esse si presentano in forma velata, pervasiva ed insidiosa e spesso sorgono all'interno dei media.

Le condizioni di mercato hanno messo a repentaglio i modelli imprenditoriali che si basano sul buon giornalismo e rafforzato quelli che considerano i media uno strumento per trarre profitto o influenze politiche. I tagli all'editoria mettono in pericolo l'industria del giornalismo. Il giornalismoinchiesta si riduce e le voci delle comunità marginalizzate e vulnerabili trovano meno ascolto.

Il mondo del giornalismo è pieno di "forze oscure": persone che fanno affari con gli inserzionisti pubblicitari per fornire materiale a pagamento camuffato da notizie vere, reporter che accettano tangenti oppure una moltitudine di pratiche losche che vengono mantenute nascoste al pubblico. Certamente molti giornalisti e molti media fanno un lavoro onesto ma, in tempi di crisi finanziaria, molti media di notizie tagliano, anche nelle democrazie europee più consolidate, alcune rubriche e infrangono i loro principi etici. In tutti i Paesi vi sono persone informate dei fatti che sanno benissimo cosa sta accadendo, ma troppo spesso sono restie a parlarne liberamente. L'Ethical Journalism Network ha aperto un dibattito su queste problematiche pubblicando, nel marzo del 2015, un report dal titolo Un- 
told stories: how corruption and conflicts of interest stalk the newsroom (White 2015b).

Il report, che riguarda 18 Paesi, inclusi gli Stati dei Balcani occidentali, il Regno Unito, l'Ucraina, la Danimarca e la Turchia, spiega come i media dell'informazione scossi dal punto di vista finanziario vengono sopraffatti da gruppi di interesse politici e aziendali. Ha rilevato che i manager del settore fanno affari con gli inserzionisti pubblicitari per fornire materiali a pagamento camuffato da notizie reali. Alcuni reporter e caporedattori accettano tangenti e pagamenti irregolari, e vi è una cultura della dipendenza da amicizie politiche ed aziendali che rende sempre più difficile distinguere il giornalismo dalla propaganda e la narrativa imparziale dalle pubbliche relazioni.

Per contrastare questa crisi, il report reclama nuove regole sulla trasparenza, sui conflitti di interesse e sulla governance etica. Tali regole sono richieste con urgenza. Molti media non osservano questi principi perché si trovano ad affrontare sfide di natura economica, ma l'indebolimento della fiducia del pubblico nel giornalismo rappresenta una conseguenza inevitabile dell'incapacità di implementare sistemi di governance interna nei media volti a eliminare i conflitti di interesse e la corruzione.

Nonostante il report faccia presente che la maggior parte delle minacce provenga da ambienti esterni ai media, e sono i governi, i politici senza scrupoli ed i comunicatori aziendali a dettare sempre più le agende mediatiche e traggono vantaggio dall'indebolimento delle redazioni tramite i tagli e le riorganizzazioni dell'economia dei media, esso mette anche in chiaro che molte ferite sono autocagionate. Evidenzia che molti proprietari mediatici di oggi non investono nel giornalismo per ragioni commerciali, bensì, nella maggioranza dei casi, per promuovere i loro affari e la loro agenda politica.

Il report rivela anche come il giornalismo è compromesso dai politici e dai proprietari nei Paesi in cui i media sono in prima linea in aspre battaglie politiche, per esempio in Turchia, ed evidenzia come, in Ucraina, la pratica del giornalismo a pagamento sia uno strumento regolarmente utilizzato da parte dei politici nei periodi che precedono le elezioni.

Le lotte che affrontano i giornalisti nelle democrazie consolidate, per esempio nel Regno Unito, non sono di minore importanza. La crescente tensione tra i gli interessi editoriali e commerciali è emersa a Londra nel febbraio del 2015, quando Peter Oborne, uno dei giornalisti politici più influenti della Gran Bretagna, si licenziò in modo drammatico ed improvviso dal quotidiano The Daily Telegraph. Oborne accusò la dirigenza di aver censurato alcuni articoli sulla banca HSBC, un'inserzionista abituale del 
giornale, coinvolto in uno scandalo fiscale internazionale. Raccontò ciò pubblicando la storia su un sito di notizie di attualità (Oborne 2015).

L'incidente evidenzia il motivo per il quale i media che valorizzano il proprio marchio e che vogliono mantenere la fiducia del pubblicano debbano disporre di controlli interni trasparenti ed affidabili per affrontare $i$ potenziali conflitti di interesse. Oborne sta ora facendo appello per una revisione delle regole sulla governance dei media e per un nuovo dibattito sull'indipendenza editoriale della stampa britannica. Il suo caso evidenzia le difficoltà nel mantenere il giornalismo etico in carreggiata nel precario mondo dei media digitali.

Il report rivela anche come in una serie di Paesi dei Balcani occidentali, segnati da una storia comune e dolorosa, la corruzione mediatica stronchi qualsiasi tentativo di liberarsi dall'ingombrante eredità della guerra, della censura e del controllo politico dei decenni del comunismo.

Il controllo del governo sulle lucrative inserzioni di Stato, le quali vengono spesso pubblicate sui media secondo le inclinazioni politiche di questi ultimi, rimane diffuso. Allo stesso tempo, l'eliminazione, in gran parte dei Paesi, della barriera invisibile che separa l'ambito editoriale da quello pubblicitario ha creato una specie di "inserzioni native", inserzioni nascoste e giornalismo a pagamento.

Nonostante le minacce maggiori derivino da fuori, dai governi, dai politici senza scrupoli e dallo strapotere delle aziende, molte ferite sono autocagionate. Il report fa riferimento ad una crescente cultura della dipendenza sul potere politico e imprenditoriale. Alcuni proprietari mediatici hanno una loro agenda imprenditoriale e politica, e molti giornalisti e caporedattori seguono pratiche redazionali che incoraggiano un giornalismo poco etico.

Ovunque si assiste ad una lotta in salita. La corruzione ed il cinismo all'interno delle redazioni portano (...), a una diminuzione dello status del lavoro giornalistico e una mancanza pervasiva di trasparenza sulle inserzioni pubblicitarie, sulla proprietà e sulle affiliazioni aziendali e politiche.

Tutto ciò richiede ai giornalisti, agli editori, ai caporedattori ed a chiunque valorizzi il pluralismo dell'informazione un compito importante: ravvivare l'impegno verso la trasparenza, la buona governance e il giornalismo etico.

Questa sfida amplifica i seri moniti espressi da Tim Berners-Lee, l'inventore del world wide web, il quale disse, nel settembre del 2014, che internet è sopraffatto dal potere dell'economia e dello Stato (Jeffries 2014). Il suo appello verso una nuova "Magna Carta" per proteggere internet è rafforzato dalla presenza di forme plurali e pulite del giornalismo in un ambiente internet sicuro. 
Nel tentativo di fornire alcune indicazioni agli operatori mediatici, il report Untold Stories (Ethical Journalism Network 2015) indica una serie di proposte riassunte in una c.d. "Agenda for Change". Questo piano in otto punti offre una serie di atti pratici per promuovere il dialogo all'interno dei media e per porre in essere alcune strutture richieste con urgenza per implementare i livelli di governance mediatica.

1. Sono richiesti un impegno significativo verso la trasparenza all'interno dei media e la pubblicazione di informazioni rilevanti relative agli interessi politici e finanziari dei proprietari, dirigenti, caporedattori e di tutti i giornalisti e presentatori rilevanti.

2. Le regole andrebbero adottate al fine di proibire le interferenze illegittime nel lavoro dei giornalisti e dei media da parte dei governi e delle istituzioni dello Stato e per stabilire i principi per la completa rivelazione dei contatti e delle transazioni tra i media ed i funzionari dello Stato.

3. Andrebbero concordati degli standard in merito alla pubblicazione di qualsiasi forma di inserzioni pubbliche e politiche e vi dovrebbe essere una regolare rivelazione dei pagamenti effettuati a tutti i giornalisti e i media per i servizi svolti.

4. Andrebbero creati sistemi indipendenti e trasparenti per valutare la circolazione e gli indici dei media.

5. Andrebbero introdotti sistemi interni per rivelare i potenziali conflitti di interessi a tutti i livelli - sia nella dirigenza, sia nelle redazioni - e formate strutture per affrontare le lamentele.

6. I contratti e le condizioni di lavoro dei giornalisti dovrebbero soddisfare gli standard lavorativi internazionali e dare ai dipendenti il diritto, senza timore di ritorsioni, di rifiutare qualsiasi forma di lavoro che violi i loro codici professionali o la loro coscienza.

7. Le regole e le procedure interne dovrebbero essere condivise in tutte le case editoriali per assicurare la rivelazione piena di tutti i contenuti a pagamento e i materiali in questione dovrebbero essere chiaramente distinguibili dal lavoro editoriale e giornalistico.

8. Andrebbero avviati dibattiti urgenti a livello nazionale e internazionale sulla necessità della creazione di strutture che forniscano assistenza pubblica per incoraggiare la previsione di un giornalismo plurale ed etico senza minare l'indipendenza editoriale. 


\section{LE SFIDE ETICHE: IL GIORNALISMO E INTERNET}

Più di quindici anni fa il redattore del Daily Mail, Paul Dacre, uno dei grandi oppositori all'interno del giornalismo britannico, disse ai sui collaboratori durante l'annuale festa d'estate del giornale: "Molti dicono che internet rappresenterà il futuro per i giornali. Bene, io dico: fesserie punto com." (The Guardian 2008).

Da allora molto è cambiato nel mondo del giornalismo, in particolare per Dacre e per i suoi colleghi. Il loro Mail Online è oggi il sito internet di informazioni di un quotidiano con il maggiore numero di accessi al mondo. Questo sito web, in cui predominano il gossip e le immagini più che lunghi reportage, non soddisferà i gusti di tutti, ma costituisce una parte importante del mix tra internet ed i media che ha trasformato in maniera profonda ed irreversibile la natura del giornalismo e la sua etica.

Oggigiorno, una nuova generazione dei media in Europa sta lavorando in collaborazione con il proprio pubblico in un ambiente informativo da poco ripulito. Coinvolge i professionisti della vecchia scuola come Dacre, i whistleblower, gli attivisti ed individui impegnati che utilizzano i social network, piccoli blog, il giornalismo dei dati ed una serie di strumenti digitali per sviluppare nuovi metodi per ricercare e per presentare le notizie. Ma, nel mezzo di questi cambiamenti redazionali, gli editori affrontano una dura battaglia per portare vita in un mercato mediatico depresso dove le notizie di rado garantiscono introiti tali da poter sostenere un giornalismo di alta qualità, e ciò include, all'apice della piramide, anche i media dell'informazione.

The Guardian, per esempio, è il terzo sito per accessi al mondo ed un leader acclamato del giornalismo di qualità, ma deve convivere con perdite dolorose di più di 100 milioni di sterline dal 2010, nonostante abbia fornito notizie di grande rilevanza pubblica, incluso lo scandalo di hackeraggio dei cellulari di News of the World e gli articoli su Edward Snowden e WikiLeaks. The Guardian e altri lottano per mantenere e per sviluppare contenuti di alta qualità all'interno di una nuova cultura del business digitale che molti ritengono profondamente immorale.

Nel suo libro Free ride, uscito nel 2011, Robert Levine lamenta che qualsiasi impresa del settore mediatico deve confrontarsi con la crescente richiesta dei consumatori di contenuti online gratuiti. Come attualmente disposto sia dal punto di vista tecnico che giuridico, scrive Levine, internet consente alle aziende informatiche di ridurre i prezzi dei contenuti a zero consentendo loro di fare affari utilizzando i contenuti protetti da copyright altrui. Fornendo contenuti per i quali queste aziende non pagano, i vettori dell'informazione e dell'intrattenimento, quali YouTube e The Huffington 
Post, diventano dei "parassiti" delle aziende mediatiche le quali investono cifre sostanziose nei giornalisti, nei musicisti e negli attori (Levine 2011).

Di conseguenza, non sorprende che molti giornalisti, caporedattori ed editori europei abbiano attaccato i giganti di internet - Facebook, Google, Apple e Amazon -, i quali sfruttano i contenuti mediatici utilizzando la tecnologia al fine di incassare gli introiti pubblicitari. Queste dispute hanno portato a conflitti di alto profilo tra imprenditori e Google, per esempio. Quest'ultima è stata accusata di costruire quasi un monopolio del mercato delle ricerche on-line in Europa utilizzando contenuti informativi dei provider tradizionali a titolo gratuito. Alcune dispute recenti con gli editori dell'informazione in Francia e, in particolare, un accordo dell'aprile del 2015 secondo il quale Google finanzierà con 150 milioni di euro progetti giornalistici di una serie di media dell'informazione europei evidenzia quanto siano divenuti delicati i rapporti in quest'area (BBC News 2015).

Il forte cambiamento nei rapporti di potere tra gli ambiti editoriali e commerciali ha creato pressioni intense sul giornalismo indipendente. Internet ci ha dato un accesso incredibile al sapere. Ha anche creato uno tsunami di informazioni che ci raggiungono da ogni dove. Vi è una raffica di informazioni in tempo reale con milioni di frammenti di informazioni. Più che mai i giornalisti servono per stabilire ciò che è importante, porre le domande giuste, inserire le informazioni nel contesto adeguato e presentare le notizie con un certo stile. Vi è una maggiore condivisione informale delle informazioni e maggiore networking. Internet sta creando degli spazi in cui molte tematiche ignorate dai media tradizionali stanno ricevendo attenzione. In Europa alcuni siti web - per esempio Stop Fake News in Ucraina - stanno fornendo un'alternativa inestimabile alle fonti informative classiche mettendo in luce come l'integrità editoriale venga minacciata dalle intromissioni politiche e dalla disinformazione. ${ }^{6}$

Allo stesso tempo vi sono più fonti informative on-line che combattono per assicurare che le opinioni di minoranza e le opinioni altrui estromesse da un settore mediatico volto al guadagno ricevano maggiore attenzione. In ogni caso, queste iniziative positive non costituiscono un'alternativa allo sviluppo di una nuova cultura etica all'interno dei media dell'informazione esistenti. Internet offre nuove opportunità per una rinascita del giornalismo trasparente e responsabile, tuttavia esse si concretizzeranno soltanto se i professionisti dell'informazione comprenderanno appieno che l'etica deve essere coltivata e la professione del giornalismo rafforzata.

6 Vedi www.stopfake.org/en/news/, consultato il 16 luglio 2015. 
L'esigenza di comunicazioni etiche viene sempre più condivisa nel mondo di internet, dove l'abuso del diritto alla libera espressione sta diventando sempre più una problematica per gli utenti ed i governi, molti dei quali sono pericolosamente inclini a imporre limitazioni giuridiche alle pubbliche comunicazioni nel nome dell'antiterrorismo, della sicurezza nazionale, del benessere pubblico o della protezione degli individui.

Il giornalismo veramente etico può fungere da fonte d'ispirazione per le comunicazioni responsabili e contrastare le peggiori manifestazioni di discorsi di odio on-line, voyeurismo, narcisismo e pregiudizi.

Andrew Keen, un veterano della Silicon Valley, ha spiegato queste minacce nel suo libro The internet is not the answer (Keen 2015). Keen è uno di molti autori che hanno preso di mira una cultura dell'informazione basata sul web che ci rende più connessi, ma meno consapevoli del mondo che ci circonda. Si tratta di un problema ben descritto da Ethan Zuckerman, il direttore del Massachussets Institute of Technology Center for Civic Media e cofondatore del sito di blogger internazionale Global Voice.

Nel suo libro Rewire: digital cosmopolitans in the age of connection Zuckerman ritiene che internet ci abbia resi tutti meno dipendenti dai giornalisti e dai caporedattori professionisti per ottenere informazioni. Le persone cercano sempre di più informazioni sui social network o utilizzando i motori di ricerca on-line (Zuckerman 2013).

Questo ha un prezzo: siamo ora esposti a tutto ciò che vogliamo sapere nella misura in cui lo dobbiamo sapere. Una cosa che aveva reso utili le forme del giornalismo tradizionale consisteva nel fatto che esse ci presentavano informazioni utili, al di fuori della nostra zona confort e su tematiche di cui sapevamo poco. Gli affari esteri, i conflitti politici e religiosi e le esperienze di altre comunità e culture possono rivelarsi cruciali per comprendere. $\mathrm{Ma}$ in un mondo determinato da strutture informative fai da te in cui il pubblico interesse viene definito come ciò che interessa al pubblico, vi è il pericolo che il web porti ad un aumento dell'ignoranza e dell'autoreferenzialità a discapito del pluralismo e di altri principi democratici ad esso legati. Il giornalismo offre l'opportunità di contrastare tale pericolo concentrandosi su ciò che i reporter sanno fare meglio: narrare storie interessanti, rilevanti, eleganti dal punto di vista stilistico e, non da ultimo, affidabili. 
9. LE SFIDE ETICHE: I BIG DATA, LA VERIFICAZIONE, IL PLAGIO E LA CORSA ALLA PUBBLICAZIONE

Mentre i principi etici dei media sono stati definiti e sostenuti per decenni, il contesto in cui essi vengono realizzati è mutato. Una delle sfide maggiori è rappresentata dallo sviluppo del giornalismo dei dati, ovvero l'uso della tecnologia per un'analisi rapida e mirata dell'informazione pubblica esistente in vaste quantità su internet.

Questa ricerca e disponibilità di enormi quantità di dati sta cambiando le vite delle persone. Ma fornisce, al contempo, più opportunità ad altri soggetti per mettere in pratica forme di sorveglianza e solleva, di conseguenza, questioni etiche per i reporter e per i caporedattori.

In Sud Africa, il giornalismo dei dati del quotidiano Ziwaphi nel distetto di Nkomazi sta migliorando le vite delle persone nell'epicentro della crisi provocata dall'Aids. La crisi si è acuita a causa dell'acqua contaminata da liquami. Il giornale ha sviluppato un modo di utilizzare i vecchi smartphone per aiutare i residenti ad ottenere acqua sicura. I telefoni vengono immersi all'interno di bottiglie di plastica e scattano foto microscopiche dell'acqua, le quali possono essere confrontate alle immagini detenute da un database pubblico al fine di individuare i livelli di Escherichia coli. I risultati vengono poi inviati ai residenti via SMS, informandoli sui luoghi in cui è possibile attingere acque sicure.

In Kenya, una stazione radio ha creato Star Health, uno strumentario per aiutare gli ascoltatori a valutare preventivamente i dottori accedendo digitalmente ai dati sugli specialisti. Aiutano le persone ad analizzare i dati per isolare e mostrare i medici fasulli ed altre forme di mala pratica (per esempio, un signore che lavorava come dottore si è rivelato essere un veterinario). Questa democraticizzazione dell'informazione è utile soltanto se i dati originari sono affidabili. I giornalisti devono essere ben consapevoli della privacy delle persone e delle problematiche legate alla sicurezza, così come dei semplici obblighi etici quali l'accuratezza e l'equità della narrazione. Non basta acquisire le competenze tecniche per trasformare i meri dati in giornalismo. Questo processo deve svolgersi in modo tale da tutelare e da informare il pubblico al quale i dati fanno riferimento.

Jeff Sonderman del Poynter Institute è convinto che i caporedattori debbano porre una serie di questioni base: "Non si creda che i dati siano naturalmente accurati, equi ed obiettivi. Non ritenere il tuo accesso ai dati o il tuo diritto di pubblicarli una ragione legittima. Rifletti in maniera critica sul bene pubblico e sui potenziali danni, sul contesto che circonda i dati e sulla sua rilevanza per il resto dei tuoi articoli. Poi decidi se la pubblicazio- 
ne dei dati di cui disponi può essere definita una forma di giornalismo" (Sonderman citato in Howard 2013).

Da nessun'altra parte la questione dei potenziali danni è stata maggiore di quando WikiLeaks ha divulgato, tra il 2010 e il 2011, a molti media dell'informazione una serie di dati provenienti dal Dipartimento della difesa degli Stati Uniti e dal Dipartimento di Stato o quando alcuni giornalisti dei quotidiani The Guardian e The Washington Post presero la dura decisione editoriale di pubblicare i documenti forniti dal collaboratore della $\mathrm{Na}$ tional Security Agency Edward Snowden. Questi casi hanno sollevato enormi questioni di pubblico interesse: il diritto alla privacy, il ruolo dello Stato, sia a livello nazionale che internazionale, nel porre in essere atti di sorveglianza e la responsabilità dei media e dei giornalisti di non rilevare informazioni che potrebbero mettere in pericolo la vita degli altri o pregiudicare la sicurezza nazionale.

In entrambi i casi, i caporedattori ed i reporter che trattavano queste notizie dovettero prendere decisioni difficili in merito alle informazioni da pubblicare. Sapevano che vi erano vite umane in gioco.

La sensibilità dell'argomento in questione e la portata globale delle informazioni pubblicate creò un contesto che non poteva essere ignorato. Alla fine essi hanno riconosciuto che si trattava di questioni troppo grandi per essere trattate da un solo organo di stampa, e optarono per una collaborazione.

Secondo il Tow Center for Digital Journalism, il modo in cui queste notizie vennero trattate, in modo associato e con la protezione dei dati, fornisce un precedente importante per il giornalismo e le generazioni dei media del futuro.

Nel migliore dei modi, come nel caso in questione, l'uso di internet, rafforzato dai valori etici, rappresenta un terreno di gioco pieno di momenti di ispirazione e di intrattenimento.

Tuttavia, ha anche un lato oscuro ove si concludono affari loschi, si scambiano immagini sporche e dove i bulli ed i bigotti corrono liberamente.

I giornalisti non sono osservatori o supervisori del comportamento umano, non è questo la loro missione. Ma dovrebbero usare cautela nel trattare le fonti digitali. La malizia ed un approccio ingannevole nei confronti della verità sono onnipresenti. Un organo di notizie affidabile deve verificare i fatti, controllare le fonti informative e tenersi alla larga dai pericolosi pettegolezzi e dalla corsa alla pubblicazione. Nel mondo della manipolazione digitale le immagini non sono sempre ciò che sembrano e non tutto ciò che una persona dice, indipendentemente da quanto ciò sia oltraggioso, è degno di pubblicazione. Si tratta di un compito arduo nella 
migliore delle ipotesi, che diventa doppiamente difficile quando la peculiarità delle comunicazioni moderne è la rapidità. I media che non vedono l'ora di battere i loro rivali internet ricercando click che generano entrate, potrebbero essere pronti a sacrificare il tempo che serve ai giornalisti per verificare i fatti ed assicurare che le loro storie sono eticamente corrette e bilanciate. Si tratta di una strategia pericolosa: la velocità non batte mai la qualità, ed anche i media migliori possono finire in situazioni di imbarazzo se adottano un approccio troppo casuale nei confronti delle fonti e delle informazioni on-line.

A volte, ovviamente, in momenti di emergenza e di crisi, i giornalisti devono fornire le informazioni che hanno ottenuto, purché diano un adeguato contesto alle questioni infervorate dalle speculazioni internet. Ma quando i media pubblicano contenuti non verificati, essi devono pubblicare, al contempo, un'"avvertenza" che spieghi da dove provengono le informazioni, il motivo per il quale potrebbero non essere affidabili e, in un secondo momento, correggere gli errori non appena diventano noti.

Un aiuto è disponibile. L'European Journalism Centre ha preparato un manuale di verificazione utile che contiene una serie di linee guida passodopo-passo su come utilizzare i contenuti creati dagli utenti nelle situazioni di emergenza. Azzeccare la storia è stata anche la mission di Storyful, un'agenzia di notizie sui social media di Dublino che aiuta i media a verificare le fonti delle notizie. L'azienda ebbe un successo tale da essere stata rilevata dal colosso dell'informazione di Rupert Murdoch.

Assicurare che il materiale sia originale, così come la protezione del $\mathrm{co}^{-}$ pyright e delle fonti, è essenziale quando si scaricano liberamente informazioni, e la mancata conoscenza delle fonti sta diventando una pratica accettata. Il plagio è sempre stato un problema per il giornalismo. Ora internet ha creato una pandemia del copia-e-incolla. Il pubblico dovrebbe sempre essere informato sulle fonti. L'unica eccezione è concessa quando vi è un interesse pubblico schiacciante o se sussistono ragioni professionali per non rivelare una fonte. Si ricordi che il giornalismo si contraddistingue per la trasparenza, la divulgazione e l'affidabilità.

Allo stesso tempo, i redattori devono affrontare lo spinoso problema delle comunicazioni on-line anonime e specialmente il comportamento sconsiderato dei c.d. "internet trolls", ovvero persone che aggrediscono altre persone in forma anonima ed ingiuriosa. Affrontare soggetti del genere è difficile perché il pubblico è stato invitato a commentare il nostro lavoro. Può essere lucrativo, generare una quantità più elevata di visualizzazioni ed indirizzare l'attenzione delle inserzioni guidate dagli algoritmi. L'opportunità di commentare il lavoro giornalistico apre inoltre le porte ad un dibat- 
tito proficuo. Ma se tutto ciò non avviene in forma controllata, può semplicemente dare adito a violenze, pregiudizi ed odio.

Molti media disattivano la sezione comenti su questioni che possono provocare odio ed intolleranza. Alcuni argomenti, per esempio il conflitto arabo-israeliano, possono essere semplicemente troppo controversi. Ma mantenere il controllo non è facile. Il numero di commenti può, talvolta, essere troppo grande per essere monitorato. Alcuni social network, per esempio Facebook, non consentono neppure ai media di bloccare i commenti. Adottare un solo standard etico in tutti i media dell'informazione è un'ambizione legittima, ma spesso poco realistica.

Il caso di Delfi in Estonia, dove un'azienda è stata condannata in giudizio per la propria incapacità di monitorare e di controllare i commenti diffamatori ed offensivi, una decisione confermata in maniera controversa dalla Corte europea dei diritti dell'uomo, dimostra che le zone di controllo editoriale dei commenti siano divenute un campo minato per i media che vogliono aumentare la propria visibilità (nella speranza di ottenere inserzioni lucrative tramite sistemi automatizzati).

Le comunicazioni pubbliche irresponsabili rese da persone che superano il limite della libera espressione ed i commenti forti, talora offensivi e che si permettono di rendere discorsi di odio o argomentazioni che incitano alla violenza, rappresentano una sfida per il giornalismo, ma anche una sfida ancor più ampia per i policy maker pubblici alla ricerca di un equilibrio tra il diritto alla libera espressione e la protezione dei diritti altrui. I media daranno il loro contribuito positivo se cercheranno di applicare regole ferree in merito a commenti ed anonimità. Linee guida chiare aiuteranno il pubblico a rispettare i valori del giornalismo. Aumentare la capacità di moderare i dibattiti on-line può costare molto dal punto di vista economico. Ma rimane comunque necessario.

\section{LE SFIDE ETICHE: I DISCORSI DI ODIO E IL TERRORISMO}

In tempi di crisi e di tensioni, è facile per i giornalisti presentare luoghi comuni casuali e reagire in modo sconsiderato a questioni che richiedono una forte e sensibile attenzione. Le guerre dell'informazione che si combattono la Russia e l'Ucraina, per esempio, si basano sulla propaganda e sulla manipolazione dei messaggi mediatici per soddisfare le faziosità politiche e non hanno nessun riguardo delle complesse realtà storiche delle due comunità coinvolte.

In tutta Europa, nel mezzo del timore pubblico sul terrorismo e sul fenomeno migratorio, vi è un crescente numero di voci da parte di politici 
senza scrupoli ed altri per generare un forte sentimento di odio, se non addirittura per incitare alla violenza.

Si tratta di un'epoca pericolosa per i media ed i giornalisti. L'esigenza di una narrazione cauta e sensibile, che i giornalisti ed i caporedattori siano "astuti" e che evitino di aumentare le tensioni tra le comunità nell'epoca dell'austerità e delle ansie, non è mai stata maggiore.

Quando vennero uccisi i giornalisti di Charlie Hebdo a Parigi, i media in Europa si unirono in solidarietà per denunciare gli omicidi. Nulla potrebbe giustificare l'assassinio di giornalisti, indipendentemente da quanto altre persone possano ritenere provocatorie le loro azioni. Ma gli stessi media - in ogni caso circa il $95 \%$ di essi - quando dovettero scegliere se riprodurre la controversa copertina di Charlie Hebdo una settimana dopo gli omicidi, si dimostrarono anche solidali nei confronti dei principi del giornalismo e molti di loro scelsero liberamente di non pubblicare la copertina oppure scelsero di farlo in forma meno dirompente. The Guardian, per esempio, pubblicò sul proprio sito web un'illustrazione in formato francobollo della pagine e avvertì i lettori. Questo approccio fu, come evidenziò l'ex caporedattore Alan Rusbridger, non una decisione che rispecchiava la solidarietà con le vittime della violenza, ma una scelta editoriale fatta sulla base di una riflessione dei valori del giornale e del rispetto per il pubblico.

Questa esigenza di un forte giornalismo etico è importante in un'epoca in cui i media possono facilmente incorrere nell'errore di dare spazio alle esternazioni oltraggianti e le azioni di determinati soggetti senza pensare alle conseguenze di ciò. Si prenda, per esempio, il caso di Terry Jones, un cristiano evangelico sconosciuto, proveniente dalla periferia della Florida rurale, che divenne di colpo una sensazione mediatica globale nel 2010 semplicemente annunciando di voler bruciare il corano. Nessuno lo avrebbe notato, o nemmeno preso in considerazione, se la sua storia non fosse stata raccolta da un giornalista locale e amplificata dalle agenzie di stampa internazionali e dalla circolazione on-line in forma virale.

I media che si affrettarono a pubblicare tale provocazione si accorsero poi di aver sbagliato a dare tanta attenzione ad un bigotto. Infatti, la sua storia non avrebbe mai dovuto dominare l'agenda mediatica, ma in questo caso la gara alla pubblicazione ed una narrazione mediatica faziosa suscettibile ai messaggi islamofobici ha provocato sommosse e vittime in Medio Oriente. Sarebbe stato possibile evitare tutto ciò se i giornalisti si fossero presi il tempo di riflettere sul contesto della storia.

Singoli atti di oltraggio estremista possono essere tranquillamente ignorati. Ciò non può dirsi però per la violenza di alto profilo e le sofisticate tecniche di propaganda digitale utilizzate da gruppi organizzati di terroristi. Utilizzando fotocamere all'avanguardia e microfoni attaccati ai loro ve- 
stiti, gli estremisti sono in grado di produrre immagini precise, di alta qualità e spessi scioccanti della loro attività omicida. Esse vengono caricate su internet in modo da essere pubblicate sui social network nella speranza di scioccare o di influenzare l'opinione pubblica oppure sui propri siti internet conosciuti solo dai sostenitori impegnati con lo scopo di fortificare la morale e reclutare nuovi adepti.

Grazie alle moderne tecnologie, i terroristi sono in grado di filmare in diretta i loro atti ottenendo la possibilità di diffondere propaganda in tempo reale e sul posto. Tra i pionieri di tali tecniche vi furono i militanti iracheni appartenenti a gruppi collegati agli attacchi contro Charlie Hebdo. Nello scorso anno, i militanti in Egitto, a Gaza, in Iraq e in Siria hanno tutti trasmesso immagini violente del loro lavoro. Video propagandistici preparati con precisione da parte di gruppi terroristici quali l'ISIS (Stato Islamico dell'Iraq e della Siria) vengono trasmessi via social network e ripresi dai media di mainstream - nonostante facciano parte di un arsenale sviluppato dai militanti al fine di diffondere i loro messaggi. Le esecuzioni trasmesse degli ostaggi dell'ISIS hanno posto problematiche scomode sui contenuti da mostrare (oppure no) al pubblico.

Mentre la maggior parte dei media sceglie di non mostrare le immagini esplicite della violenza terroristica, i media potrebbero comunque chiedersi se essi non vengano sedotti troppo facilmente dalla propaganda terroristica di alta qualità.

Affrontare i discorsi di odio sta diventando più difficile a causa della mancanza di una definizione chiara e facilmente comprensibile di ciò che sono i discorsi d'odio. I giornalisti vengono spesso accusati di discorsi d'odio, ed infatti alcuni commentatori utilizzano parole consapevolmente provocatorie e violente quando sta loro bene, ma nella stragrande maggioranza dei casi i giornalisti ed i media si rendono colpevoli soltanto di riportare le mere esternazioni altrui.

Nel tentativo di aiutare i media, l'Ethical Journalism Network ha sviluppato una semplice valutazione, per i giornalisti, consistente in cinque punti. Evidenzia le questioni che i giornalisti e i caporedattori dovrebbero porre nel decidere se utilizzare materiali editoriali controversi:

\subsection{Quali sono la posizione o lo status dell'autore del messaggio?}

I giornalisti ed i caporedattori devono capire che il mero fatto che una persona dica qualcosa di offensivo non crea una notizia. I giornalisti devono, infatti, tenere conto del contesto in cui l'affermazione viene resa e lo status e la reputazione dell'autore del messaggio. Ad un politico demagogico 
esperto nel manipolare il pubblico non dovrebbe essere prestata attenzione mediatica soltanto perché egli/ella crea un clima negativo o rende dichiarazioni non comprovate o controverse. Ciò che viene detto deve essere inserito e riportato nel contesto adeguato.

Quando persone che non sono personaggi pubblici emettono esclamazioni d'odio, potrebbe essere saggio ignorarle del tutto. La libertà di parola è un diritto che appartiene a chiunque, inclusi i politici e i personaggi pubblici, ed è compito del giornalista assicurare che ognuno possa esprimersi, ma ciò non significa accordare un diritto alla menzogna, alla diffusione di dicerie maliziose $\mathrm{o}$ all'incitamento all'ostilità $\mathrm{o}$ alla violenza contro un particolare gruppo. Quando le persone parlano a vanvera, il buon giornalismo dovrebbe mantenere dritta la barra per tutti.

\subsection{Qual è la portata del discorso?}

Una conversazione privata svoltasi in un luogo pubblico può includere le opinioni più indicibili, ma causa un danno piuttosto contenuto e, di conseguenza, non violerebbe la valutazione dei discorsi d'odio. Tuttavia, la situazione cambia se il discorso viene diffuso attraverso i media più comuni o su internet. I giornalisti devono altresì tenere in considerazione la frequenza e l'estensione della comunicazione - si tratta di un'esplosione di invettive e di odio breve, momentanea e stemperata, oppure la dichiarazione viene resa in forma voluta e continua? È una questione di comportamento o si tratta di un incidente singolo? La ripetizione rappresenta un indicatore utile per capire se si tratta di una strategia intenzionale per scatenare un sentimento di ostilità nei confronti di altre persone.

\subsection{Quali sono le intenzioni dell'autore?}

Solitamente i giornalisti etici ed i caporedattori ben informati sono in grado di comprendere rapidamente se il discorso è volutamente finalizzato ad attaccare o a mettere a repentaglio i diritti altrui. Come parti del processo di narrazione i giornalisti ed i caporedattori hanno la particolare responsabilità di inserire il discorso nel contesto adeguato per rivelare e riportare gli obiettivi dell'autore. Non è nostra intenzione rivelare o attaccare le persone con cui ci troviamo in disaccordo, ma una narrazione attenta ed etica aiuta sempre le persone a comprendere meglio il contesto in cui una determinata esternazione viene resa. 
Vanno poste alcune questioni chiave: in che modo giova all'autore ed agli interessi che egli o ella rappresenta? Quali sono le vittime del messaggio e qual è l'impatto su di loro, sia come individui sia all'interno della comunità?

\subsection{Quali sono il contenuto e la forma del messaggio?}

I giornalisti devono giudicare se il messaggio è provocatorio e diretto, in quale forma è stato prodotto e lo stile in cui è stato reso. C'è una differenza abissale tra un discorso fatto al bar davanti ad un gruppo ristretto ed un'orazione pubblica davanti ad una folla impressionata.

Molte persone hanno idee ed opinioni offensive. Non è un reato, e non è un reato rendere tali opinioni pubbliche (le persone lo fanno di continuo su internet e sui social), ma le parole e le immagini che adoperano possono sortire effetti devastanti se incitano alla violenza.

I giornalisti dovrebbero porsi le seguenti questioni: questo discorso/ questa esternazione sono pericolose? Possono causare conseguenze giuridiche? Inciteranno alla violenza o promuoveranno un aumento del rancore nei confronti di terzi?

\subsection{Qual è il clima economico, sociale e politico?}

I messaggi pericolosi o controversi nascono soprattutto in tempi di austerità e di tensioni sociali, quando le paure del pubblico sono molte e quando i politici si combattono a vicenda. I giornalisti devono tenere conto dell'aria che tira nel momento in cui il discorso viene fatto. Una campagna elettorale infiammata in cui i gruppi politici sgomitano per ottenere l'attenzione pubblica offre spesso lo sfondo per commenti al vetriolo. I giornalisti devono valutare se l'esternazione è equa, se si basa su fatti reali e se è ragionevole nelle circostanze date.

I giornalisti devono essere soprattutto attenti. È importante che essi prendano in considerazione determinati aspetti: qual è l'impatto del messaggio sulle persone direttamente coinvolte? Sono esse in grado di ricevere il messaggio in condizioni di relativa sicurezza? L'esternazione punta a peggiorare o a migliorare lo stato dei fatti? Chi è colpito in senso negativo dal messaggio in questione? 
CONCLUSIONE: UN NUOVO AMBIENTE PER LE POLITICHE NEL SETTORE DEI MEDIA E L'ETICA

Nonostante la moltitudine di sfide indicate nel presente saggio, sussistono molte ragioni per pensare in modo positivo, se non addirittura ottimistico.

Le nuove fonti dell'informazione aprono le porte ad un giornalismo più inclusivo, ad un pluralismo più ampio e ad un panorama di opinioni e commenti pubblici molto più vasto. La tecnologia ha concesso la libertà di espressione a milioni di persone che non la possedevano prima. Le persone possono dire qualsiasi cosa vogliano, indipendentemente da quanto vergognoso o ammirevole ciò sia, e fare ciò ogniqualvolta lo desiderino.

Ma una maggiore sfida in questo nuovo mondo delle comunicazioni aperte è data dall'esigenza di promuovere standard di responsabilità nel modo in cui noi tutti utilizziamo le informazioni, per dare un senso a queste nuove libertà.

Sostenere, in linea di principio, i valori qui enunciati non è sufficiente. $\mathrm{Al}$ fine di creare un ambiente in cui tutti - $\mathrm{i}$ giornalisti e la comunità più ampia inclusi - siano in grado di trarre beneficio da una società dell'informazione aperta, è necessario analizzare l'ambiente in cui il giornalismo viene praticato per stabilire ciò che va cambiato ed adottare una serie di priorità che creeranno un nuovo panorama dell'informazione in Europa.

Esiste, ovviamente, un ruolo importante che devono ricoprire i governi e servono politiche sui media ufficiali per sostenere questa difficile sfida. Quattro tematiche vengono qui brevemente illustrate:

- Gli Stati membri del Consiglio d'Europa dovrebbero offrire un sostegno costituzionale alla libertà di espressione, e laddove devono porre limiti a tale libertà, deve trattarsi di limitazioni ben definite e sostenute dal punto di vista giuridico. Per esempio, le leggi in materia di diffamazione e calunnia, una piaga per il giornalismo in molti Paesi, dovrebbero essere depenalizzate e si dovrebbe porre fine alle sanzioni esageratamente elevate nelle cause civili che riguardano i media.

- Una preoccupazione chiave dei governi consiste nel combattere la minaccia terroristica in Europa, e gli eventi recenti in Europa, non da ultimo lo spaventoso assassinio di 17 persone a Parigi nel gennaio del 2015, di cui 12 negli uffici di Charlie Hebdo. Ciò ha portato alla minaccia di nuove leggi riguardanti la libera espressione a sostegno di movimenti radicali ed estremisti. Sussiste, inoltre, una crescente preoccupazione pubblica circa la tendenza verso un aumento della sorveglianza dei cittadini. Entrambi questi sviluppi potrebbero ledere l'esercizio del gior- 
nalismo ed i governi devono essere cauti prima di emanare nuove leggi che potrebbero indebolire le libertà civili.

- Allo stesso tempo, i governi dovrebbero sostenere il principio di autoregolamentazione e incoraggiare i media a sviluppare sistemi di autoregolamentazione credibili ed effettivi basati su un codice etico condiviso dai professionisti dei media. Dovrebbero esserci dei meccanismi per ricevere e rispondere alle lamentele e sostegni alle iniziative per promuovere la responsabilità dei media tramite un consiglio dei media o tramite un garante locale.

- Ma soprattutto, i governi e le autorità a livello nazionale e locale dovrebbero aprirsi alle critiche del pubblico. Gli Stati possono sostenere gli sforzi dei giornalisti e dei media per soddisfare il diritto del pubblico a sapere, rispondendo in maniera immediata e costruttiva alle richieste dei giornalisti. Dovrebbero esistere leggi che prevedono un diritto di accesso alle informazioni detenute dagli enti pubblici quanto più ampio possibile con, allo stesso tempo, poche eccezioni definite nel dettaglio che potrebbero includere il settore del welfare pubblico, ragioni di sicurezza e la moralità individuale.

Queste politiche sono essenziali per sostenere la creazione di un ambiente in cui ci sia spazio per costruire una nuova partnership tra i media ed il pubblico finalizzata allo sviluppo di una cultura della responsabilità nelle comunicazioni. Per fare ciò, è essenziale che il mestiere del giornalismo venga sostenuto all'interno e al di fuori delle redazioni. Sappiamo già che il pubblico punirà ciò che considera standard inaccettabili del giornalismo.

Come affermato da John Birt, l'ex Direttore generale della BBC, se il giornalismo si mostra arrogante, "il pubblico non si batterà per lui" (Birt, citato in Hargreaves 2003: 266). La BBC, la quale cerca di sostenere gli standard del giornalismo, ha sofferto a causa dei propri errori così come $\mathrm{i}$ tycoon dei media che hanno approfittato del giornalismo tabloid. Rupert Murdoch, forse il più potente imprenditore nel settore mediatico della fine del Novecento, venne costretto dallo sdegno pubblico a chiudere il giornale News of the World nel Regno Unito, indebolito dallo scandalo dei telefoni cellulari spiati. Questi incidenti confermano soprattutto che il giornalismo non è soltanto una questione d'affari, ma un servizio con un obiettivo delineato da una serie di valori.

Troppo spesso i proprietari ed i caporedattori dei media tendono a ignorare questa realtà. In futuro, se vorranno costruire la fiducia del pubblico, dovranno essere più energici nel difendere il giornalismo e nel sostenere $\mathrm{i}$ media trasparenti ed affidabili rinforzati da redazioni che sono vani moto- 
re di una narrazione accurata e bilanciata. Possono fare ciò collaborando con i propri giornalisti e con il pubblico.

Il futuro del giornalismo in questo panorama frammentato caratterizzato da comunicazioni pubbliche più ampie e più flessibili dipenderà dalla sua capacità di soddisfare gli interessi pubblici e di nutrire la cornice etica all'interno della quale le notizie vengono riportate.

Il pubblico dei media, ora divenuto un partner nella ricerca delle informazioni, pretende ostinatamente che i media mantengano le lori promesse in termini di trasparenza, responsabilità e buona pratica e, nell'affrontare queste sfide, i giornalisti possono porsi in una posizione tale da fornire una leadership in un dibattito rinnovato su etica e libertà di espressione.

Ciò che è bene per il giornalismo è bene anche per gli altri. Sicuramente i valori del giornalismo illustrati nel presente saggio - l'accuratezza, l'indipendenza, l'imparzialità, l'umanità e la responsabilità - non possono essere applicati in tutto il panorama dell'informazione pubblica.

Ciò sarebbe assurdo e sfiderebbe il diritto al trattare questioni autoreferenziali che è un punto centrale della concezione di libertà di espressione.

In ogni caso, alcuni di questi valori giornalistici possono essere applicati universalmente. Anche il più fazioso dei comunicatori del mondo della politica, della religione, dell'impresa o della semplice autopromozione dovrebbe ritenere accettabile che, una volta entrati nell'ambito dell'informazione pubblica, le comunicazioni dovrebbero attenersi ai fatti, mostrare umanità e rispetto per il prossimo ed essere responsabili in caso di errori (correggendo le informazioni false). Si tratta di valori chiave che affondano le loro radici nel giornalismo etico e che dovrebbero essere il fondamento di qualsiasi teoria sui media e politica dell'informazione. Dato un sostengo adeguato, essi possono ispirare una nuova era di comunicazioni responsabili, non soltanto in Europa, bensì in tutto il mondo.

Aidan White è il Direttore dell'Ethical Journalism Network. Per 24 anni è stato il Segretarioo generale della Federazione internazionale dei giornalisti e il primo Segretario generale della Federazione europea dei giornalisti.

\section{BIBLIOGRAFIA}

BBC News (2015), "Google offers cash support to Europe's news groups", BBC News Services, 27 aprile 2015, disponibile su www.bbc.co.uk/news/technology-3 2486019, consultato il 16 luglio 2015.

Blum D. (2013), President Barack Obama, the Kindle Singles interview, disponibile su www. mediabistro.com/galleycat/president-barack-obama-interviewed-in-freekin dle-single_b75128, consultato il 16 luglio 2015 
Davies N. (2008), Flat Earth news, Chatto and Windus.

Ethical Journalism Network (2015), "Media standards audit: reporting for good governance and transparency in Pakistan", disponibile su http://ethicaljournalis mnetwork.org/en/contents/media-standards-audit-reporting-for-good-governanc e-andtransparency-in-pakistan, consultato il 16 luglio 2015.

Ethical Journalism Network (2015), "Untold Stories: How Corruption and Conflicts of Interest Stalk the Newsroom”, disponibile su http://ethicaljournalismnet work.org/en/contents/untold-stories-how-corruption-and-conflicts-of-interest-stal k-the-newsroom, consultato il 30 luglio 2015.

Hargreaves I. (2003), Journalism: truth or dare, Oxford University Press.

Howard A. (2013), "On the ethics of data-driven journalism: of fact, friction and public records in a more transparent age", Tow Center for Digital Journalism, 14 novembre 2013, disponibile su http://towcenter.org/on-the-ethics-of-data-driv en-journalism-offact-friction-and-public-records-in-a-more-transparent-age/, consultato il 17 luglio 2015.

Human Rights Watch (2011), "Hungary: media law endangers press freedom”, 7 gennaio 2011, disponibile su www.hrw.org/news/2011/01/07/hungary-media-la w-endangers-press-freedom, consultato il 16 luglio 2015.

Jeffries S. (2014), "How the web lost its way - and its founding principles", The Guardian, 24 agosto 2014, disponibile su www.theguardian.com/technology/201 4/aug/24/internet-lost-its-way-tim-berners-lee-world-wide-web, consultato il 16 luglio 2015.

Keen A. (2015), The Internet is not the answer, Atlantic Books, London.

Lambeth E. B. (1992), Committed journalism, an ethic for the profession, 2a ed., Indiana University Press, Bloomington, Indiana.

Levine R. (2011), Free ride: how the Internet is destroying the culture business and how the culture business can fight back, Bodley Head.

Myers M. (2012), "Is there a link between media and good governance? What the academics say", a report to the Center for International Media Assistance, disponibile su www.cima.ned.org/wp-content/uploads/2015/02/Myers-Mary-Goo d-Governance-Academics-Survey-07-06-12-FINAL.pdf

Oborne P. (2015), "Why I have resigned from The Telegraph", Our Kingdom, 17 febbraio 2015, disponibile su www.opendemocracy.net/ourkingdom/peter-obor ne/why-i-haveresigned-from-telegraph, consultato il 16 luglio 2015.

O’Neill O. (2004), "Rethinking freedom of the press", Reith Lecture, Royal Irish Academy, 4 dicembre 2004.

Silverman C. (a cura di) (2014), Verification handbook: a definitive guide to verifying digital content for emergency coverage, European Journalism Centre, disponibile su http://verificationhandbook.com/, consultato il 17 luglio 2015.

The Guardian (2008), Media top 100 2008, 14 luglio 2008, disponibile su www.theg uardian.com/media/2008/jul/14/mediatop10020082, consultato il 30 luglio 2015. 
The Guardian (2015), Charlie Hebdo: first cover since terror attack depicts prophet $M u$ hammad, 13 gennaio 2015, disponibile su www.theguardian.com/media/2015/ja n/13/charlie-hebdo-cover-magazine-prophet-muhammad, consultato il 30 luglio 2015.

White A. (ed.) (2015a), The trust factor: an EJN review of journalism and self-regulation, Ethical Journalism Network, Londra, disponibile su http://ethicaljournalis mnetwork.org/en/contents/the-trust-factor-an-ejn-review-of-journalism-and-self-r egulation, consultato il 16 luglio 2015.

White A. (ed.) (2015b), Untold stories: how corruption and conflicts of interest stalk the newsroom, Ethical Journalism Network, Londra, disponibile su http://ethicaljour nalismnetwork. org/en/contents/untold-stories-how-corruption-and-conflicts-ofintereststalk-the-newsroom, consultato il 16 luglio 2015.

Zuckerman (2013), Rewire: digital cosmopolitans in the age of connection, W. W. Norton \& Company Inc., New York. 
